\title{
Complex-conjugate-resolved imaging using two-harmonic FD-OCT
}

\author{
Andrei B. Vakhtin*, Kristen A. Peterson, and Daniel J. Kane \\ Southwest Sciences, Inc., 1570 Pacheco St., Suite E-11, Santa Fe, NM, USA 87505
}

\begin{abstract}
The two-harmonic FD-OCT method, where the quadrature components of the spectral interferogram are obtained by simultaneous acquisition of the first and second harmonics of the phase-modulated interferogram, is used for complexconjugate-resolved imaging of biological samples. The method is implemented using sampling of the phase modulated interferogram with an integrating detector array followed by digital demodulation at the first and second harmonics. A complex conjugate rejection ratio as high as $70 \mathrm{~dB}$ is achieved.
\end{abstract}

Keywords: optical coherence tomography; medical and biological imaging; interferometry

\section{INTRODUCTION}

The major drawback of FD-OCT that limits its practical use is the complex conjugate ambiguity, which is inherent to real Fourier transforms. Because of this artifact, an FD-OCT image obtained from a real valued spectral interferogram consists of two overlapped images symmetrical with respect to the zero plane (zero phase delay) of the interferometer. To avoid ambiguity in interpretation of the image, the zero plane must be positioned outside of the imaged sample. Thus, only one half of the imaging depth range is useful in practice. Resolving the complex conjugate ambiguity would double the imaging depth range and provide additional flexibility by allowing arbitrary positioning of the zero plane, e.g., moving it inside the sample.

The problems of obtaining the full complex interferogram and resolving the complex conjugate ambiguity in FD-OCT have been addressed using different approaches, including phase-shifting methods, ${ }^{1,2}$ simultaneous detection of the quadrature components of the interferogram by using $3 \times 3$ fiber-optic couplers, ${ }^{3-5}$ separation of the two complex conjugate images using carrier frequency shifting in swept-source FD-OCT, ${ }^{6-8}$ and using polarization-based optical demodulation. ${ }^{9}$ Recently we presented a new method of direct acquisition of the complex spectral interferogram, where the imaginary and real components are detected simultaneously as the first and second harmonics of a phase-modulated interferogram. ${ }^{10}$ The technique is applicable to both swept-source FD-OCT and broadband/parallel FD-OCT. The twoharmonic method provides efficient suppression of the complex conjugate artifact (as well as the dc and autocorrelation artifacts) and has the following advantages over the existing full-range FD-OCT techniques: (1) Since the phase is modulated with a pure sine wave, the requirements to the phase modulating and signal acquisition electronics are not very demanding (only ac signals at the first and second harmonics of the phase modulation frequency have to be detected); (2) The real and imaginary parts of the spectral interferogram are acquired simultaneously using the same detector; (3) For a given imaging rate, the detection bandwidth for harmonic detection is no greater than that required for standard parallel or swept source FD-OCT; therefore no reduction in the signal-to-noise ratio is expected.

While implementation of the two-harmonic method in swept-source FD-OCT appears straightforward, the broadband/parallel FD-OCT will require special hardware, i.e., a demodulating detector array. In this work, as the first step to parallel implementation of the two-harmonic FD-OCT, we use sampling of the phase modulated spectral interferogram with an InGaAs integrating detector array followed by digital demodulation at the first and second harmonics. The two-harmonic FD-OCT images of a Xenopus Tropicalis tadpole show the complex conjugate artifact rejection ratio as high as $70 \mathrm{~dB}$. Also, we demonstrate high-speed two-harmonic FD-OCT data acquisition using a silicon line-scan camera.

*avakhtin@swsciences.com; phone 1505 984-1322; fax 1505 988-9230

Coherence Domain Optical Methods and Optical Coherence Tomography in Biomedicine XI edited by James G. Fujimoto, Joseph A. Izatt, Valery V. Tuchin

Proc. of SPIE Vol. 6429, 64291A, (2007) -1605-7422/07/\$18 - doi: 10.1117/12.701020

Proc. of SPIE Vol. 6429 64291A-1 


\section{METHODOLOGY}

\subsection{Experimental}

A Michelson-type fiber optic interferometer is constructed using a 50/50 coupler and single mode fibers. A Superlum BroadLighter 1300 SLD (center wavelength $1300 \mathrm{~nm}$, FWHM $85 \mathrm{~nm}$ ) is used as a light source. A sine wave is applied to the piezo-translator holding the reference mirror to phase modulate the interferometer at a frequency of $23 \mathrm{~Hz}$. The phase modulated spectral interferograms are sampled at a rate of $200 \mathrm{~Hz}$ using an InGaAs integrating array detector (Hamamatsu G9202-512S, 16-bit digitization) coupled to a spectrograph (Oriel 77200, 1/4 m, grating $600 \mathrm{l} / \mathrm{mm}$ ). Normally, a block of 400 spectral interferograms, 512 spectral points each, and covering a wavelength range of 1220$1375 \mathrm{~nm}$ are acquired at a rate of $200 \mathrm{~Hz}$, which takes $2 \mathrm{~s}$. The block of phase modulated spectra is then resampled to equal spacing in optical frequency, digitally demodulated at the first and second harmonics of the modulation frequency, and low-pass filtered (time constant $1 \mathrm{~s}$ ) to yield the real and imaginary components of the complex spectral interferogram (see Sec 2.2). The inverse Fourier transform applied to the complex spectral interferogram produces a full-range A-scan.

To demonstrate fast two-harmonic FD-OCT data acquisition, a different setup is used, where a Ti:sapphire laser (center wavelength $795 \mathrm{~nm}$, FWHM $27 \mathrm{~nm}$ ) and a line-scan camera (Atmel AViiVA M2CL1014, 12-bit digitization) are used as the light source and the detector, respectively. Also, a free-space Michelson interferometer is used instead of the fiber interferometer. The phase is modulated at $5.95 \mathrm{kHz}$, and a block of 800 spectral interferograms, 1024 spectral points each, covering a wavelength range of $722-864 \mathrm{~nm}$, are acquired at a rate of $47.6 \mathrm{kHz}$. In this experiment, data acquisition and data processing are performed separately; real-time data processing has not been implemented yet. The block of phase modulated spectra is converted to equally spaced optical frequency domain and digitally demodulated at the first and second harmonics of the modulation frequency. The digital demodulation procedure is described in Sec 3.

\subsection{Two-harmonic FD-OCT}

For simplicity consider FD-OCT imaging of a sample that has only one back-scattering surface (e.g., a mirror). The spectral interferogram at the output of a phase modulated interferometer can be expressed as follows:

$$
I_{\mathrm{SI}}(\omega, t)=I_{\mathrm{R}}(\omega)+I_{\mathrm{S}}(\omega)+2\left[I_{\mathrm{R}}(\omega) I_{\mathrm{S}}(\omega)\right]^{1 / 2} \cos \left[\Delta \phi_{\mathrm{S}}(\omega)+\phi_{0}(\omega, t)\right]
$$

where $\omega$ is the optical frequency, $t$ is time; $I_{\mathrm{R}}(\omega)$ and $I_{\mathrm{S}}(\omega)$ are the light intensities in the reference and the sample arms of the interferometer, respectively; $\Delta \phi_{\mathrm{S}}(\omega)$ is the phase delay corresponding to the sample; $\phi_{0}(\omega, t)$ is time-dependent phase term that describes phase modulation. With harmonic phase modulation at a frequency $f$, the latter term becomes

$$
\phi_{0}(\omega, t)=a_{\mathrm{m}}(\omega) \sin \omega_{\mathrm{m}} t,
$$

where $\omega_{\mathrm{m}}=2 \pi f$, and $a_{\mathrm{m}}(\omega)$ is the phase modulation amplitude. Expanding the last term on the right side of Eq. (1) into series of Bessel functions $\left(J_{0}, J_{1}, J_{2}, \ldots\right)$ we have

$$
\begin{gathered}
I_{\mathrm{SI}}(\omega, t)=I_{\mathrm{R}}(\omega)+I_{\mathrm{S}}(\omega)+2\left[I_{\mathrm{R}}(\omega) I_{\mathrm{S}}(\omega)\right]^{1 / 2}\left\{J_{0}\left[a_{\mathrm{m}}(\omega)\right]-2 J_{1}\left[a_{\mathrm{m}}(\omega)\right] \sin \omega_{\mathrm{m}} t \sin \Delta \phi_{\mathrm{S}}(\omega)+\right. \\
\left.2 J_{2}\left[a_{\mathrm{m}}(\omega)\right] \cos 2 \omega_{\mathrm{m}} t \cos \Delta \phi_{\mathrm{S}}(\omega)-2 J_{3}\left[a_{\mathrm{m}}(\omega)\right] \sin 3 \omega_{\mathrm{m}} t \sin \Delta \phi_{\mathrm{S}}(\omega)+2 J_{4}\left[a_{\mathrm{m}}(\omega)\right] \cos 4 \omega_{\mathrm{m}} t \cos \Delta \phi_{\mathrm{S}}(\omega)-\ldots\right\} .
\end{gathered}
$$

As follows from Eq. (3), the signals demodulated at the first $\left(H_{1}\right)$ and the second $\left(H_{2}\right)$ harmonics of the modulation frequency are

$$
\begin{aligned}
& H_{1}\left[\omega, \Delta \phi_{\mathrm{S}}(\omega)\right]=-4 J_{1}\left[a_{\mathrm{m}}(\omega)\right]\left[I_{\mathrm{R}}(\omega) I_{\mathrm{S}}(\omega)\right]^{1 / 2} \sin \Delta \phi_{\mathrm{S}}(\omega) \\
& H_{2}\left[\omega, \Delta \phi_{\mathrm{S}}(\omega)\right]=4 J_{2}\left[a_{\mathrm{m}}(\omega)\right]\left[I_{\mathrm{R}}(\omega) I_{\mathrm{S}}(\omega)\right]^{1 / 2} \cos \Delta \phi_{\mathrm{S}}(\omega) .
\end{aligned}
$$

$H_{1}$ and $H_{2}$ (as any other pair of odd and even harmonics) represent the sine and cosine, i.e., imaginary and real parts of the complex spectral interferogram, respectively. The complex inverse Fourier transform can be applied to the complex interferogram to convert it into the time domain profile that is free from complex conjugate ambiguity and also the dc and autocorrelation terms:

$$
f(\tau)=\mathfrak{I}^{-1}\left\{\beta H_{2}\left[\omega, \Delta \phi_{\mathrm{S}}(\omega)\right]-i H_{1}\left[\omega, \Delta \phi_{\mathrm{S}}(\omega)\right]\right\} .
$$

According to Eqs (4) and (5), the scaling coefficient $\beta$ is equal to

$$
\beta=J_{1}\left[a_{\mathrm{m}}(\omega)\right] / J_{2}\left[a_{\mathrm{m}}(\omega)\right]
$$


and depends only on the modulation amplitude, which is, generally, wavelength dependent. For example, if the phase modulation is achieved by dithering the reference mirror, $a_{\mathrm{m}}$ scales linearly with the optical frequency $\omega$, which can easily be taken into account during signal acquisition and signal processing. The modulation amplitude $a_{\mathrm{m}}$ should be chosen so that the zeros of the first- and second-order Bessel functions $J_{1}$ and $J_{2}$ are avoided, and the first- and secondharmonic signals are comparable in magnitude. The practically useful range is $0.8 \leq a_{\mathrm{m}} / \mathrm{rad} \leq 3.5$.

\section{RESULTS AND DISCUSSION}

Figure 1 shows two-harmonic FD-OCT cross-sectional images of a Xenopus Tropicalis stage 45 tadpole acquired using a $1300 \mathrm{~nm}$ SLD and an InGaAs integrating array detector. The images shown on the left and right panels are obtained using the full complex spectral interferograms and only the real parts of the interferograms, respectively. The eyes, optic nerves, brain, and other features of the internal structure of the animal are clearly visible. The images in Fig. 1 show that the two-harmonic FD-OCT method can produce clean rejection of the complex conjugate artifact. Normally, the complex conjugate image is suppressed to the background noise level and is not visible at all. The only exception is the very bright image of the eye on the right side of the image, which produces a relatively strong complex conjugate artifact. Figure 2 shows representative examples of A-scans that correspond to that area (indicated by the arrows in Fig. 1). The rejection ratio is typically $50-60 \mathrm{~dB}$, and can be as low as $40 \mathrm{~dB}$ and as high as $70 \mathrm{~dB}$. The reasons for incomplete rejection, as well as the presence of the residual dc artifact at the zero phase delay are not completely understood; they may be attributed to the slow data acquisition, which is sensitive to the low-frequency phase noise inherent to the fiber-optic interferometer.
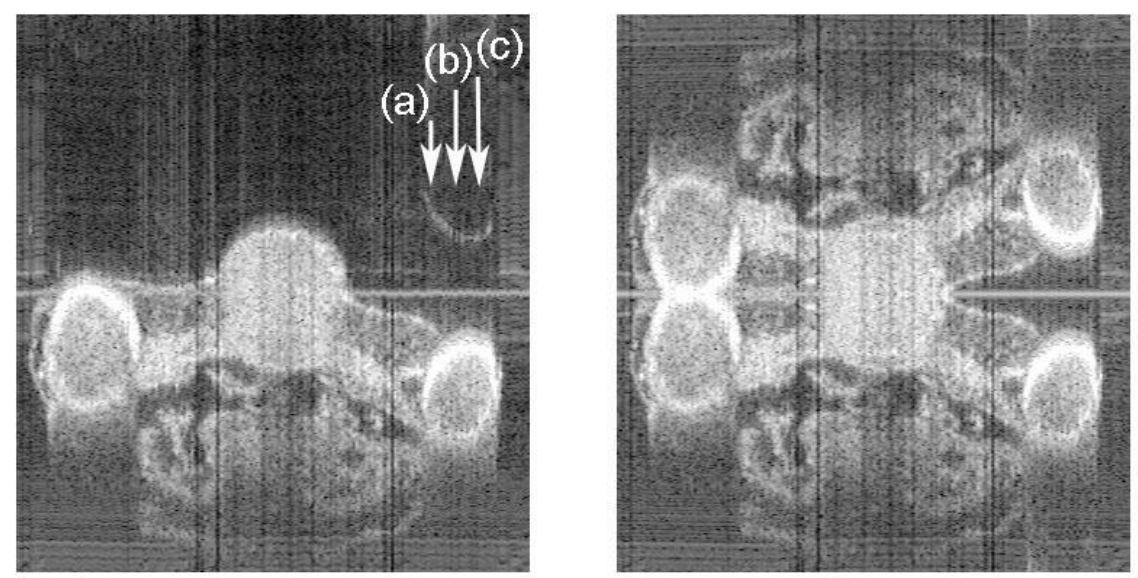

Fig. 1. FD-OCT cross-sectional transverse images from within the head of a stage 45 Xenopus Tropicalis tadpole (dorsal surface) obtained from complex spectral interferograms (left panel) and the real parts of the spectral interferograms (right panel). Modulation amplitude $a_{\mathrm{m}}=2.77 \mathrm{rad}$. Arrows labeled (a), (b), and (c) indicate A-scans shown in Fig. 2.
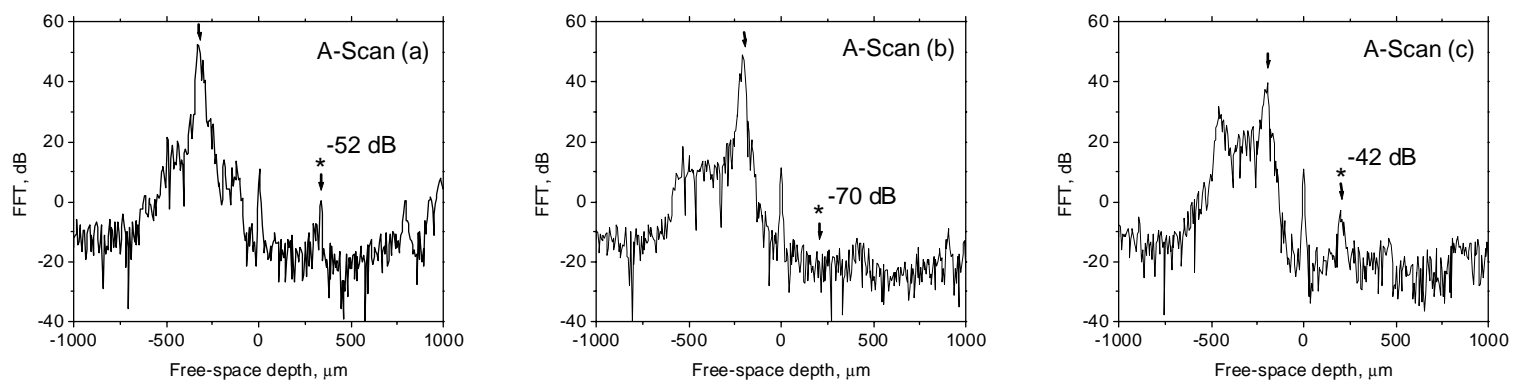

Fig. 2. A-scans indicated in Fig. 1. On each plot, arrows indicate the strongest peak and its complex conjugate. The complex conjugate rejection ratios are listed. Residual dc peaks are visible at zero depth. 
High-speed two-harmonic FD-OCT data acquisition is demonstrated using a silicon line-scan camera. Figure 3 shows FD-OCT images of a mirror obtained using the complex (Figs. 3a-c) and real (Figs. 3d-f) spectral interferograms, respectively. In this experiment the phase is modulated at $5.95 \mathrm{kHz}\left(a_{\mathrm{m}}=1.85 \mathrm{rad}\right)$, and the spectra are sampled at 47.6 $\mathrm{kHz}$, i.e. exactly eight spectra are collected during one phase modulation period. Digital demodulation of the time dependent signals acquired at each spectral point is performed by multiplying the signal by $\sin \omega_{\mathrm{m}} t$ and $\sin 2 \omega_{\mathrm{m}} t$ waves (with phases optimized to obtain maximum in-phase signals) followed by integration over $8 n$ data points, where $n$ is the number of modulation periods, over which the integration is performed. By varying $n$ within 1-100 we found that, although large $n$ is helpful in increasing the signal-to-noise ratio by averaging the signal over multiple modulation periods, rejection of the complex conjugate artifact remains good even with $n=1$, i.e., when the signal is demodulated over just one phase modulation period. As an illustration, Figs. 3a-c show full-range FD-OCT images obtained using demodulation over 1,10 , and 100 modulation periods. With $n=1$, the complex conjugate rejection ratio of $46-62 \mathrm{~dB}$ is routinely achieved. Acquiring one A-scan at a rate of $5.95 \mathrm{kHz}$ corresponds to an imaging rate of approximately thirty $1024 \times 200$ full-range FD-OCT frames per second, which is video-rate data acquisition. Experiments on real-time data processing are in progress.

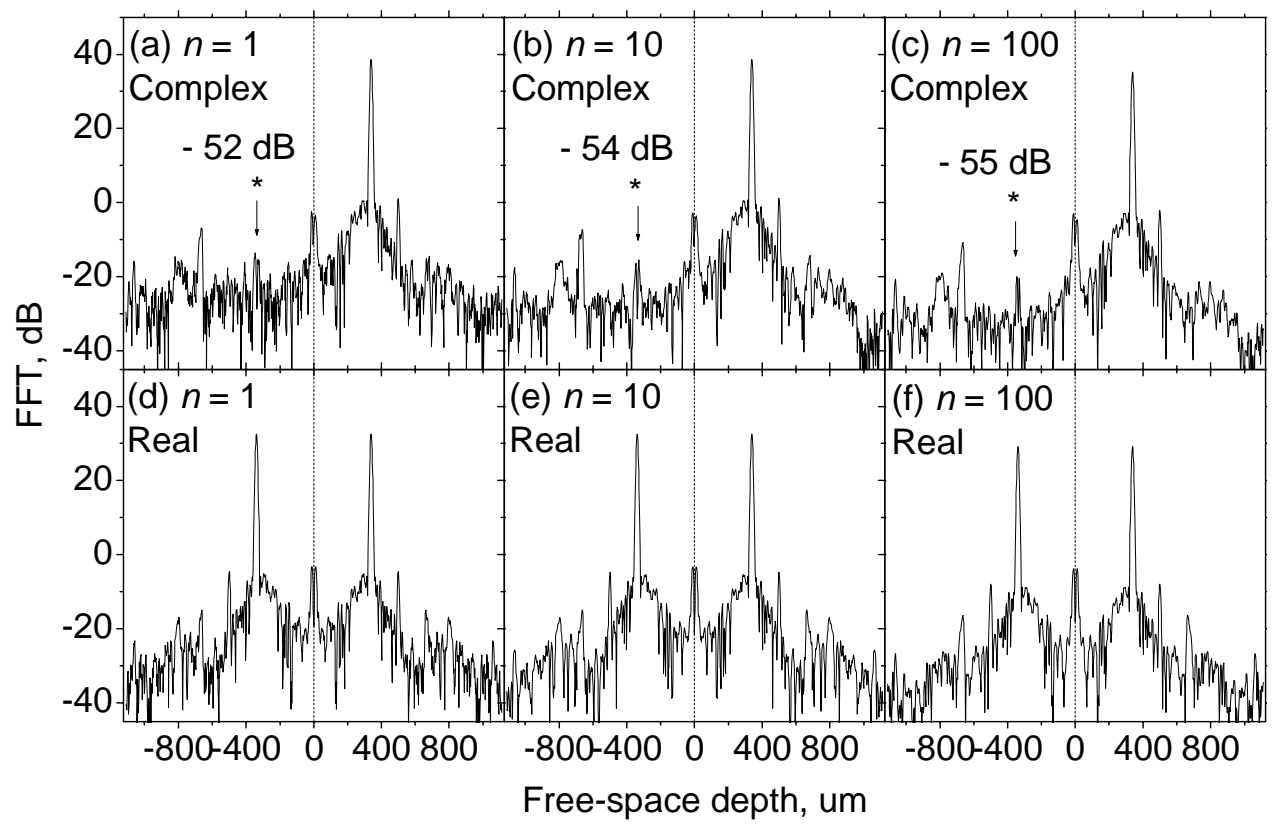

Fig. 3. Representative examples of A-scans of a mirror obtained using high-speed two-harmonic FD-OCT data acquisition (modulation amplitude $a_{\mathrm{m}}=1.85 \mathrm{rad}$ ). (a-c) A-scans obtained using the complex spectral interferograms. The number of modulation periods, $n$, over which the integration is performed, is shown on each panel. The complex artifact rejection ratios are also indicated. (d-f) The same A-scans obtained using only the real part of the spectral interferograms.

In summary, we present images of biological samples obtained using our two-harmonic complex-conjugate-resolved FD-OCT method. The technique is implemented using sampling of the phase modulated interferograms with an integrating InGaAs array detector, followed by digital demodulation. The complex conjugate rejection ratio of up to 70 $\mathrm{dB}$ is obtained with a tadpole used as a sample. Also, we demonstrate high-speed two-harmonic FD-OCT data acquisition using a silicon line-scan camera. Our experimental data show feasibility of video-rate full-range FD-OCT imaging with a complex conjugate rejection ratio of at least 46-62 dB. 


\section{REFERENCES}

1. M. Wojtkowski, A. Kowalczyk, R. Leitgeb, and A. F. Fercher, "Full range complex spectral optical coherence tomography technique in eye imaging," Opt. Lett. 27(16), 1415-1417 (2002).

2. R. A. Leitgeb, C. K. Hitzenberger, A. F. Fercher, and T. Bajraszewski, "Phase-shifting algorithm to achieve highspeed long-depth-range probing by frequency-domain optical coherence tomography," Opt. Lett. 28(22), 2201-2203 (2003).

3. M. A. Choma, C. Yang, and J. A. Izatt, "Instantaneous quadrature low-coherence interferometry with $3 \times 3$ fiberoptic couplers," Opt. Lett. 28(22), 2162-2164 (2003).

4. M. V. Sarunic, M. A. Choma, C. Yang, and J. A. Izatt, "Instantaneous complex conjugate resolved spectral domain and swept-source OCT using 3x3 fiber couplers," Opt. Express 13(3), 957-967 (2005).

5. M. V. Sarunic, B. E. Applegate, and J. A. Izatt, "Real-time quadrature projection complex conjugate resolved Fourier domain optical coherence tomography," Opt. Lett. 31(16), 2426-2428 (2006).

6. J. Zhang, J. S. Nelson, and Z. Chen, "Removal of a mirror image and enhancement of the signal-to-noise ratio in Fourier-domain optical coherence tomography using an electro-optic phase modulator," Opt. Lett. 30(2), 147-149 (2005).

7. S. H. Yun, G. J. Tearney, J. F. de Boer, and B. E. Bouma, "Removing the depth-degeneracy in optical frequency domain imaging with frequency shifting," Opt. Express 12(20), 4822-4828 (2004).

8. A. M. Davis, M. A. Choma, and J. A. Izatt, "Heterodyne swept-source optical coherence tomography for complete complex conjugate ambiguity removal," J. Biomed. Opt. 10(6), 064005 (2005).

9. B. J. Vakoc, S. H. Yun, G. J. Tearney, and B. E. Bouma, "Elimination of depth degeneracy in optical frequencydomain imaging through polarization-based optical demodulation," Opt. Lett. 31(3), 362-364 (2006).

10. A. B. Vakhtin, K. A. Peterson, and D. J. Kane, "Resolving the complex conjugate ambiguity in Fourier domain OCT by harmonic lock-in detection of the spectral interferogram," Opt. Lett. 31(9), 1271-1273 (2006). 